MATHEMATICS OF COMPUTATION

Volume 71, Number 238, Pages 767-780

S 0025-5718(01)01336-9

Article electronically published on November 14, 2001

\title{
SOME COMPUTATIONS \\ ON THE SPECTRA OF PISOT AND SALEM NUMBERS
}

\author{
PETER BORWEIN AND KEVIN G. HARE
}

\begin{abstract}
Properties of Pisot numbers have long been of interest. One line of questioning, initiated by Erdős, Joó and Komornik in 1990, is the determination of $l(q)$ for Pisot numbers $q$, where

$$
l(q)=\inf \left(|y|: y=\epsilon_{0}+\epsilon_{1} q^{1}+\cdots+\epsilon_{n} q^{n}, \epsilon_{i} \in\{ \pm 1,0\}, y \neq 0\right) .
$$

Although the quantity $l(q)$ is known for some Pisot numbers $q$, there has been no general method for computing $l(q)$. This paper gives such an algorithm. With this algorithm, some properties of $l(q)$ and its generalizations are investigated.

A related question concerns the analogy of $l(q)$, denoted $a(q)$, where the coefficients are restricted to \pm 1 ; in particular, for which non-Pisot numbers is $a(q)$ nonzero? This paper finds an infinite class of Salem numbers where $a(q) \neq 0$
\end{abstract}

\section{INTRODUCTION}

We begin by recalling the definition of a Pisot number:

Definition 1. A Pisot number is a positive real algebraic integer, all of whose conjugates are of modulus strictly less than 1. A Pisot polynomial is the minimal polynomial of a Pisot number.

The main question we address is what happens when particular classes of polynomials with restricted coefficients are evaluated at a real number. More formally:

Definition 2. Let $S$ be a finite set of integers and $q$ a real number. Define the spectrum of $q$ with respect to $S$ as

$$
\Lambda^{S}(q):=\left\{\epsilon_{0}+\epsilon_{1} q^{1}+\cdots+\epsilon_{n} q^{n}: \epsilon_{i} \in S\right\} .
$$

For convenience let $\Lambda^{m}(q):=\Lambda^{\{-m,-m+1, \ldots, m-1, m\}}(q), \Lambda(q):=\Lambda^{1}(q)$, and $A(q):=$ $\Lambda^{\{ \pm 1\}}(q)$.

One issue with respect to these spectra is to find the the minimal nonzero value.

Definition 3. Define

$$
l^{S}(q):=\inf \left(|y|: y \in \Lambda^{S}(q), y \neq 0\right),
$$

and let $l^{m}(q):=l^{\{-m,-m+1, \ldots, m-1, m\}}(q), l(q):=l^{1}(q)$ and $a(q):=l^{\{ \pm 1\}}(q)$.

Received by the editor April 12, 2000 and, in revised form, August 8, 2000.

2000 Mathematics Subject Classification. Primary 11Y60, 11 Y 40.

Key words and phrases. Pisot numbers, LLL, spectrum, beta numbers.

Research of K.G. Hare supported by MITACS and by NSERC of Canada, and P. Borwein supported by MITACS and by NSERC of Canada. 
Here and throughout $q \in(1,2)$, which is the only domain of interest for such questions.

A spectrum $\Lambda$ is discrete if, for any finite interval $[a, b]$ of the real line, $\Lambda \cap[a, b]$ has only a finite number of elements. A spectrum is uniformly discrete if there exists an $\epsilon$ greater than zero such that for any two distinct values in the spectrum, these values are at least $\epsilon$ apart. Similarly, a spectrum is nonuniformly discrete if it is discrete, but it is not uniformly discrete.

It is clear that $A(q) \subset \Lambda(q)$, so a number of the results known for $\Lambda(q)$ follow for $A(q)$. Results known for $A(q)$ specifically are due to Peres and Solomyak 18. They show that $A(q)$ is dense in $\mathbb{R}$ for a.e. $q \in(\sqrt{2}, 2)$. Further, if $q \in(1, \sqrt{2})$ and $q^{2}$ is not the root of a height 1 polynomial, then $A(q)$ is dense. Also if $q$ is a Pisot number, then $A(q)$ is uniformly discrete.

This paper answers some questions concerning $A(q)$. It gives necessary conditions for $A(q)$ to be discrete. As well it gives some examples of $q$ that are not Pisot, but where the $A(q)$ are discrete. The existence of such a $q$ is somewhat surprising, because $\Lambda(q)$ is thought to be discrete if and only if $q$ is Pisot. Further, until now the only known examples for $A(q)$ being discrete were for Pisot numbers.

If $q$ is a Pisot number, then much is known about $l^{m}(q)$. In general $l^{m}(q)$ is known to be strictly positive for all Pisot numbers $q$ and for all $m$ [3, 8]. Specifically,

$$
l(q) \geq(1+q)^{-1} q^{(\log (d-1) \log (1+q) \log (1-Q)) / \log (Q)}>0,
$$

where $d$ is the degree of the minimal polynomial satisfied by $q$, and $Q$ the modulus of $q$ 's largest conjugate [10]. For general $m$, it is known that

$$
l^{m}(q) \geq \frac{\left(1-\left|q_{2}\right|\right) \cdots\left(1-\left|q_{n}\right|\right)}{m^{n-1}}>0
$$

where $q_{2}, \ldots, q_{n}$ are the conjugates of $q\left[3\right.$, 11. The stronger result that $l^{m}(q)>0$ for all $m$ if and only if $q$ is Pisot is given in [3]. An even stronger result, shown in [6] on a more restricted set, is that if $q<\frac{1+\sqrt{5}}{2}$, then $l^{2}(q)>0$ if and only if $q$ is Pisot. However it is still unknown if there exists a $q \in(1,2)$ that is not Pisot with $l(q)>0$.

When $q$ is not a Pisot number, then the following results are known. If $q$ does not satisfy a polynomial of height 1 , then $l(q)=0$ by a pigeonhole argument [3, 10]. Some other results of Erdös and Komornik [8] include:

1. If $q$ is not Pisot and $m \geq q-q^{-1}$, then $\Lambda^{m}(q)$ has a finite accumulation point.

2. If $q$ is not Pisot, then $l^{m}(q)=0$ for all $m \geq\left\lceil q-q^{-1}\right\rceil+\lceil q-1\rceil$.

3. If $1<q \leq 2^{1 / 4}$ and if $q^{2}$ is not the first or second Pisot number, then $l^{m}(q)=0$ for all $m$.

Specific values of $l^{m}(q)$ have been calculated for some Pisot numbers $q$. If $q$ is the Pisot number that satisfies $q^{3}-q^{2}-1$, then $l(q)=q^{2}-2$ [14. If $q$ is the Pisot number satisfying $q^{n}-q^{n-1}-\cdots-1$, then $l(q)=\frac{1}{q}$ [7]. If $q$ is the Golden ratio (the greater root of $x^{2}-x-1$ ), then $l^{2}(q)=2-q[3]$. For general $m$, and $q$ the Golden ratio, all $l^{m}(q)$ are known: if $F_{k}$ is the $k$ th Fibonacci number $\left(F_{0}=0, F_{1}=\right.$ $\left.1, F_{n}=F_{n-1}+F_{n-2}\right)$ and $q^{k-2}<m \leq q^{k-1}$, then $l^{m}(q)=\left|F_{k} q-F_{k+1}\right|$ [14].

This paper gives an algorithm where $l^{m}(q)$ can be calculated for any Pisot number $q$ and any integer $m$, limited only by the memory of the computer.

An algorithm to determine $\Lambda(q) \cap\left[\frac{-1}{q-1}, \frac{1}{q-1}\right]$ is given by Ka-Sing Lau in [15]. In Lau's paper the values of the size of the spectra in this range are determined for 
the Pisot roots of the polynomials $x^{3}-x^{2}-x-1, x^{3}-2 x^{2}+x-1, x^{2}-x-1$, $x^{3}-x^{2}-1, x^{4}-x^{3}-1$ and $x^{3}-x-1$. This algorithm is generalized in Section 2 to answer some of the questions concerning $l(q)$ and $a(q)$ above.

Another question that can be examined with this algorithm asks which Pisot numbers $q$ satisfy a polynomial with \pm 1 coefficients. Surprisingly there exist Pisot numbers that do not satisfy such a polynomial. For the Pisot numbers that do satisfy a \pm 1 polynomial, it is often possible to use LLL to find this polynomial. In fact, LLL can sometimes be used for other algebraic numbers for this purpose, as will be demonstrated in Section 6. For a good review of LLL, see [4, 16, 17].

\section{The ALGORITHM}

This section gives an algorithm to find the spectrum of a number in a particular range (i.e., determine $\Lambda^{S}(q) \cap[a, b]$ for a number $q$ and a finite range $[a, b]$ ). This section shows that when this number is a Pisot number, then this algorithm terminates, and further if this algorithm terminates, then the spectrum is discrete over the entire real line. In the case of $\Lambda(q)$, this algorithm is very similar to that given by Ka-Sing Lau, [15]. First the following obvious lemma is needed:

Lemma 1. Let $S$ be a finite set of integers. Let $p(x)$ be a degree $n$ polynomial with coefficients in $S$. Let $s_{l} \leq S \leq s_{u}$ be lower and upper bounds for the integers in $S$, and let $q>1$. Denote $\alpha_{u}:=\frac{-s_{l}}{q-1}$ and $\alpha_{l}:=\frac{-s_{u}}{q-1}$. If $p(q) \notin\left[\alpha_{l}, \alpha_{u}\right]$, then $q \times p(q)+s \notin\left[\alpha_{l}, \alpha_{u}\right]$ for all $s \in S$.

From this it follows that, if $p(q) \notin\left[\alpha_{l}, \alpha_{u}\right]$, the polynomial $q \times p(q)+s$ need not be looked at, as it cannot contribute to the spectrum in this range. Further if $\alpha_{l}^{*} \leq \alpha_{l}$ and $\alpha_{u}^{*} \geq \alpha_{u}$, then the same result follows for the range $\left[\alpha_{l}^{*}, \alpha_{u}^{*}\right]$.

The next lemma will ensure that an exhaustive search for all elements in a finite range for a given spectrum will terminate if $q$ is a Pisot number.

Lemma 2. If $q$ is a Pisot number, and $S$ is a finite set of integers, then $\left|\Lambda^{S}(q) \cap[a, b]\right|$ is finite.

Proof. Let $r=\max \{|s|: s \in S\}$. Let $y_{1}, y_{2} \in \Lambda^{S}(q), y_{1} \neq y_{2}$. Then $y_{1}-y_{2} \in \Lambda^{2 r}(q)$; hence, $\left|y_{1}-y_{2}\right| \geq l^{2 r}(q)>0$, where the last inequality comes from [3, 8, 9]. Thus $\left|\Lambda^{S}(q) \cap[a, b]\right| \leq \frac{b-a}{l^{2 r}(q)}<\infty$.

Algorithm 1 is one way to write an exhaustive search to determine $\Lambda^{S}(q) \cap\left[\alpha_{l}, \alpha_{u}\right]$.

As the spectrum of the Pisot numbers is uniformly discrete (this follows from [8, 11]), then $\left|\Lambda^{S}(q) \cap\left[\alpha_{l}, \alpha_{u}\right]\right|<\infty$ for $q$ Pisot. The converse to this is

Theorem 2.1. If $\left|\Lambda^{S}(q) \cap\left[\alpha_{l}, \alpha_{u}\right]\right|<\infty$, then $\Lambda^{S}(q)$ is discrete.

Note that, in Theorem [2.1 $q$ is not assumed to be Pisot. It is worth noting that only discreteness is shown, and not uniform discreteness. In fact, there exist examples of spectra, described later in Section [5, which have provably nonuniformly discrete spectra. 


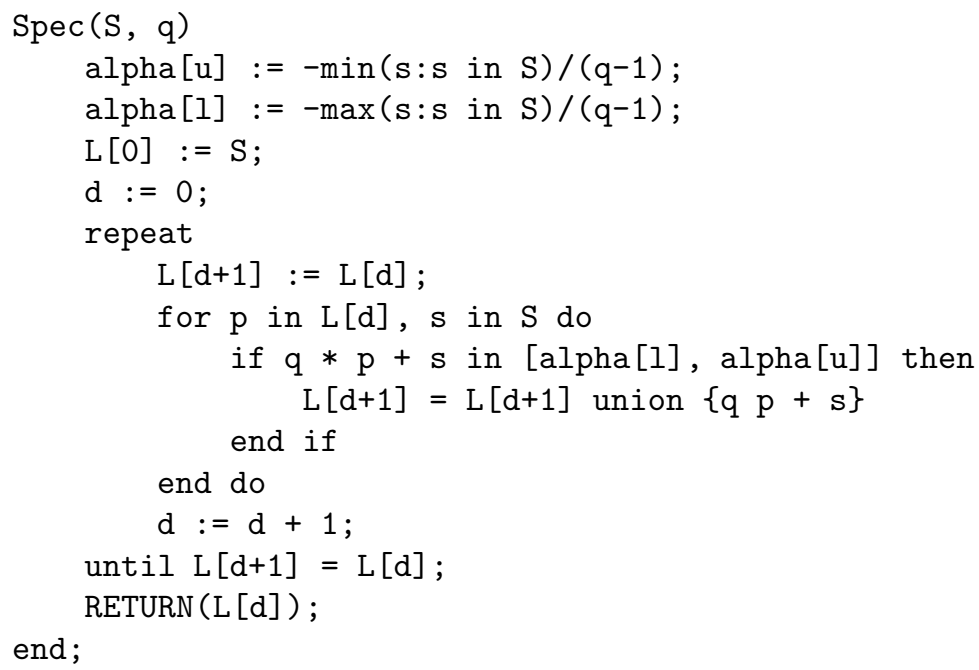

Algorithm 1: Finding the Spectrum of a Pisot number $q$.

Proof. Let $s_{l}$ and $s_{u}$ be lower and upper bounds for $S$, and $\alpha_{l}$ and $\alpha_{u}$ be as defined in Lemma 1 Define $\lambda_{1}:=\Lambda^{S}(q) \cap\left[\alpha_{l}, \alpha_{u}\right]$. Define

$$
\alpha_{u, 1}:=\min \left\{q \beta+s>\alpha_{u}: \beta \in \lambda_{1}, s \in S\right\}
$$

and define $\alpha_{l, 1}$ similarly to be the first element in the spectrum less than $\alpha_{l}$. From this, define $\alpha_{u, n}:=q \times \alpha_{u, n-1}+s_{l}$ and $\alpha_{l, n}:=q \times \alpha_{l, n-1}+s_{l}$. Further define $\lambda_{n}:=\left[\alpha_{l, n-1}, \alpha_{u, n-1}\right] \cap \Lambda^{S}(q)$.

Clearly, $\alpha_{u, n} \rightarrow \infty$ and $\alpha_{l, n} \rightarrow-\infty$ as $n \rightarrow \infty$. By assumption $\lambda_{1}$ has only a finite number of elements. Noticing that

$$
\lambda_{n}=\lambda_{n-1} \cup\left\{\left\{q \beta+s: \beta \in \lambda_{n-1}, s \in S\right\} \cap\left[\alpha_{l, n-1}, \alpha_{u, n-1}\right]\right\}
$$

gives by induction that $\lambda_{n}$ has only a finite number of elements. Thus $\Lambda^{S}(q)$ is discrete, as required.

\section{Programming tricks}

The algorithm described in Section 2 was implemented in $\mathrm{C}++$. This section will summarize some of the techniques used to improve the efficiency of this code.

When calculating $\Lambda^{S}(q)$ a list of all polynomials examined must be kept. As the degree of these polynomials can be quite large, this list of polynomials can take up a large amount of memory. To reduce the space requirements, the actual code stored the remainders of the polynomials when divided by the minimal polynomial of $q$. This is advantageous as the degree is bounded above; and through experimentation it is noted that the height of these polynomials being stored do not grow larger than a "short int" in $\mathrm{C}++$.

This has a second advantage; if $p_{1}(q)=p_{2}(q)$ where $p_{1}$ and $p_{2}$ are polynomials, then the remainders of $p_{1}$ and $p_{2}$ when divided by the minimal polynomial of $q$ are equal. Thus duplication within the spectrum is easily recognized. 
These polynomials are stored in a Red-Black tree with a lexigraphical order on the coefficients, but any height regulating tree with any order would give similar results for time comparisons of duplication recognition. For more information on Red-Black trees, see [5].

The next observation to be made is that if $S$ is symmetric (i.e., $s \in S$ implies that $-s \in S)$, then $\Lambda^{S}(q)$ is symmetric. Utilizing this symmetry eliminates half of the calculations needed.

Lastly, a technique that was considered, but decided not necessary for this preliminary investigation, allowed the problem to be partitioned up into an arbitrarily large number of subproblems. This would allow the constraining factor on the calculations to be disk space instead of RAM. Further, with a careful implementation, this technique could also be utilized to parallelize the calculation.

This technique to partition the problem up is described most easily by means of an example. Let $S=\{ \pm 1\}$ and let $P(x)=x^{3}-x-1$ be the first Pisot polynomial (with approximate root 1.324717957). Let $m=2$ (here $m$ could be chosen to be any integer). Divide the set of polynomials in the spectrum into $|P(m)|=5$ residues, depending upon their evaluation at $m$. Consider the set of polynomials with coefficients in $S$ known at some point in the calculation which evaluates at $m$ to $2(\bmod 5)$. Then these polynomials, when extended, will evaluate at $m$ to either 3 , or $0(\bmod 5)(\operatorname{as} 2 \times 2+1 \equiv 0(\bmod 5)$ and $2 \times 2-1 \equiv 3(\bmod 5))$. Thus, when the polynomials which evaluate to $2(\bmod 5)$ at $m$ are being examined, the set of polynomials of residue 1 or 4 need not be in memory. In general, at most $\frac{|S|+1}{p(m)}$ (a fraction tending to 0 as $m$ tends to infinity) of the polynomials need be in memory at any given time.

Naive methods failed to calculate $\Lambda(q)$ for 16 examples, all of degree 10. Thus it was decided that the overhead needed to implement this technique was not justified.

\section{Some questions of $\Lambda(q)$ And $A(q)$ For Pisot numbers $q$}

Algorithm 1 has been used to calculate $l(q)$ for all Pisot numbers up to and including degree 9, and $a(q)$ for Pisot numbers up to and including degree 10. (Recall, $l(q)=l^{\{ \pm 1,0\}}(q)$ and $a(q)=l^{\{ \pm 1\}}(q)$.) To determine the Pisot numbers up to and including degree 10, the methods of David Boyd were used [1]. There are 232 Pisot numbers of degree less than or equal to 10 (between 1 and 2 inclusive) and thus we do not include all of the results here. However all these results, as well as the code used to determine them, can be found at [12].

In the calculations of $a(q)$ and $l(q)$, there were only a few cases where these values coincided. Some of these are enumerated in Table 1. The polynomials associated with $l(q)$ in this table are the \pm 1 polynomial modulo the Pisot polynomial which gives $\pm l(q)$.

The number of Pisot numbers of degree less than or equal to 10 where $a(q)=l(q)$ is fairly small, and it would be interesting to know if the set of Pisot numbers with this property is finite.

The next question of interest is which Pisot numbers $q$ satisfy a \pm 1 polynomial. This is equivalent to asking if $0 \in A(q)$. This was answered in the affirmative for most Pisot numbers. The first failure is of degree 6 . It is interesting to note that all of the examples found where $0 \notin A(q)$ are such that this root $q$ is greater than 1.95. It would be interesting to know if this must always be the case. These results are summarized in Table 2 
TABle 1. Pisot numbers where $l(q)=a(q)$.

\begin{tabular}{||l|l|l|l||}
\hline Pisot polynomial & $q$ & $l(q)$ & $\begin{array}{l}\text { Polynomial associated } \\
\text { with } l(q)\end{array}$ \\
\hline$x^{2}-x-1$ & 1.618034 & 0.618034 & $x-1$ \\
$x^{3}-2 x^{2}+x-1$ & 1.754878 & 0.245122 & $x-2$ \\
$x^{3}-x^{2}-x-1$ & 1.839286 & 0.543689 & $x^{2}-x-1$ \\
$x^{4}-x^{3}-1$ & 1.380278 & 0.008993 & $x^{3}-4 x^{2}+5$ \\
$x^{4}-2 x^{3}+x-1$ & 1.866760 & 0.13324 & $x-2$ \\
$x^{4}-x^{3}-2 x^{2}+1$ & 1.905166 & 0.068706 & $x^{3}-3 x^{2}+x+2$ \\
$x^{4}-x^{3}-x^{2}-x-1$ & 1.927562 & 0.518790 & $x^{3}-x^{2}-x-1$ \\
$x^{5}-x^{4}-x^{3}+x^{2}-1$ & 1.443269 & 0.002292 & $4 x^{2}-3 x-4$ \\
$x^{5}-x^{3}-x^{2}-x-1$ & 1.534158 & 0.002155 & $2 x^{4}-3 x^{3}+x^{2}-3 x+2$ \\
$x^{5}-x^{4}-x^{2}-1$ & 1.570147 & 0.006992 & $x^{4}-2 x^{2}-2 x+2$ \\
$x^{5}-2 x^{4}+x^{3}-x^{2}+x-1$ & 1.673649 & 0.009705 & $x^{4}-x^{3}-x^{2}-2 x+3$ \\
$x^{5}-x^{4}-x^{3}-1$ & 1.704903 & 0.030844 & $2 x^{3}-3 x^{3}-2$ \\
$x^{5}-x^{4}-x^{3}-x^{2}-x-1$ & 1.965948 & 0.508660 & $x^{4}-x^{3}-x^{2}-x-1$ \\
$x^{6}-x^{5}-x^{4}+x^{2}-1$ & 1.501595 & 0.0003491 & $x^{5}+2 x^{4}-4 x^{3}-3 x^{2}+3 x-2$ \\
$x^{6}-2 x^{5}+x-1$ & 1.967168 & 0.032831 & $x-2$ \\
$x^{6}-x^{5}-x^{4}-x^{3}$ & 1.983583 & 0.504138 & $x^{5}-x^{4}-x^{3}-x^{2}$ \\
$-x^{2}-x-1$ & & & $-x-1$ \\
$x^{7}-x^{5}-x^{4}-x^{3}$ & 1.590005 & 0.0001137 & $4 x^{6}-5 x^{5}-x^{4}-x^{3}$ \\
$-x^{2}-x-1$ & & & $+x-6$ \\
$x^{7}-x^{6}-x^{4}-x^{2}-1$ & 1.601347 & 0.0004642 & $2 x^{5}-x^{4}-3 x^{3}-1 x^{2}-x+2$ \\
$x^{7}-2 x^{6}+x^{5}-x^{4}$ & 1.640728 & 0.0003030 & $2 x^{6}-2 x^{5}-2 x^{4}$ \\
$+x^{3}-x^{2}+x-1$ & & & $-2 x^{2}+x+3$ \\
$x^{7}-2 x^{6}+x^{5}-2 x^{4}$ & 1.790223 & 0.0006021 & $x^{6}-3 x^{5}+5 x^{4}-4 x^{3}$ \\
$+2 x^{3}-x^{2}+x-1$ & & & $-4 x+1$ \\
$x^{7}-2 x^{6}+x-1$ & 1.983861 & 0.016138 & $x-2$ \\
$x^{7}-x^{6}-x^{5}-x^{4}$ & 1.991964 & 0.502017 & $x^{6}-x^{5}-x^{4}-x^{3}$ \\
$-x^{3}-x^{2}-x-1$ & & & $-x^{2}-x-1$ \\
\hline \multicolumn{2}{|r|}{} & \multicolumn{2}{|c||}{} \\
\hline
\end{tabular}

TABLE 2. Pisot polynomials that do not divide a \pm 1 polynomial.

\begin{tabular}{||l|l|}
\hline Pisot polynomial & Pisot number \\
\hline$x^{6}-x^{5}-2 x^{4}+x^{2}-x-1$ & 1.979476326 \\
$x^{6}-3 x^{5}+3 x^{4}-2 x^{3}+x-1$ & 1.955451068 \\
$x^{8}-x^{7}-x^{6}-x^{5}-2 x^{4}+1$ & 1.995777793 \\
$x^{9}-x^{8}-x^{7}-2 x^{6}+x^{3}-x^{2}-x-1$ & 1.997784254 \\
$x^{9}-2 x^{8}+x^{5}-2 x^{4}+x-1$ & 1.996283920 \\
$x^{9}-x^{8}-x^{7}-x^{6}-x^{5}-x^{4}-x^{3}-x^{2}-1$ & 1.994016415 \\
$x^{9}-2 x^{7}-3 x^{6}-2 x^{5}+x^{3}-x-1$ & 1.992483962 \\
$x^{9}-x^{8}-x^{7}-x^{6}-x^{5}-x^{4}-x^{3}-x-1$ & 1.989944545 \\
$x^{9}-x^{8}-x^{7}-x^{6}-x^{5}-x^{4}+1$ & 1.963515789 \\
$x^{10}-x^{9}-x^{8}-x^{7}-x^{6}-2 x^{5}+1$ & 1.998987762 \\
$x^{10}-x^{9}-2 x^{8}+x^{6}-x^{5}-2 x^{4}+x^{2}-x-1$ & 1.998772685 \\
$x^{10}-2 x^{9}+x^{7}-2 x^{6}+x^{4}-2 x^{3}+x-1$ & 1.998277927 \\
$x^{10}-2 x^{9}+x^{5}-x^{4}-x+1$ & 1.969456013 \\
$x^{10}-x^{9}-2 x^{8}+x^{6}-x^{5}-x^{4}+x^{3}+x^{2}-x-1$ & 1.966884957 \\
$x^{10}-x^{9}-x^{8}-x^{7}-x^{6}-x^{5}+1$ & 1.964715641 \\
$x^{10}-2 x^{8}-3 x^{7}-x^{6}-x^{3}+x+1$ & 1.954062236 \\
\hline
\end{tabular}


TABLE 3. Successful calculations with a spectrum over 20 million for $l(q)$.

\begin{tabular}{|c|c|c|c|c|}
\hline Pisot polynomial & $q$ & $l(q)$ & $\begin{array}{l}\text { Approximate } \\
\text { size of } \\
\text { spectrum in } \\
{\left[\alpha_{l}, \alpha_{u}\right]}\end{array}$ & $\begin{array}{l}\mathrm{CPU} \\
\text { secs }\end{array}$ \\
\hline $\begin{array}{c}x^{10}-x^{9}-x^{8}-x^{7} \\
+x^{6}-x^{3}+1\end{array}$ & 1.742975573 & $1.18668 \mathrm{e}-07$ & 26973910 & $39 \mathrm{~m} 50 \mathrm{~s}$ \\
\hline $\begin{array}{l}x^{10}-x^{9}-x^{7}-x^{6} \\
\quad-x^{5}-x^{4}-x^{3} \\
\quad-x^{2}-1\end{array}$ & 1.746541923 & 7.04603e-08 & 41498130 & $58 \mathrm{~m} 41 \mathrm{~s}$ \\
\hline $\begin{array}{l}x^{10}-x^{9}-x^{8}-x^{7} \\
\quad+x^{5}-x^{3}+1\end{array}$ & 1.795572823 & $3.5123 \mathrm{e}-08$ & 43357472 & $1 \mathrm{~h} 1 \mathrm{~m} 7 \mathrm{~s}$ \\
\hline $\begin{array}{l}x^{10}-x^{9}-x^{8}-x^{7} \\
-x^{3}+1\end{array}$ & 1.852234868 & $8.17922 \mathrm{e}-08$ & 25981420 & $34 \mathrm{~m} 38 \mathrm{~s}$ \\
\hline $\begin{array}{c}x^{10}-x^{9}-x^{8}-x^{7} \\
-x^{5}+x^{4}+1\end{array}$ & 1.860952864 & $3.80874 \mathrm{e}-07$ & 24944436 & $35 \mathrm{~m} 22 \mathrm{~s}$ \\
\hline $\begin{array}{l}x^{10}-2 x^{9}+x^{8}-2 x^{7} \\
\quad+x^{6}+x^{3}-x^{2} \\
\quad+x-1\end{array}$ & 1.870250440 & $4.44816 \mathrm{e}-08$ & 46252634 & $1 \mathrm{~h} 4 \mathrm{~m} 56 \mathrm{~s}$ \\
\hline $\begin{array}{c}x^{10}-2 x^{9}+x^{7}-x^{6} \\
-x^{3}+x^{2}-1\end{array}$ & 1.881601063 & $2.57611 \mathrm{e}-07$ & 27513576 & $35 \mathrm{~m} 35 \mathrm{~s}$ \\
\hline $\begin{array}{c}x^{10}-2 x^{8}-3 x^{7}-x^{6} \\
+x^{5}+2 x^{4}+x^{3} \\
-x^{2}-2 x-1\end{array}$ & 1.890027098 & $2.67873 \mathrm{e}-07$ & 20923016 & $29 \mathrm{~m} 43 \mathrm{~s}$ \\
\hline $\begin{array}{l}x^{10}-2 x^{9}+x^{8}-x^{7} \\
\quad-x^{6}-x^{2}+x-1\end{array}$ & 1.903832902 & $2.22525 \mathrm{e}-07$ & 22738454 & $28 \mathrm{~m} 42 \mathrm{~s}$ \\
\hline $\begin{array}{c}x^{10}-x^{9}-x^{8}-x^{7}-x^{5} \\
-x^{4}-x^{2}-x-1\end{array}$ & 1.921407084 & $3.12296 \mathrm{e}-08$ & 41511868 & $57 \mathrm{~m} 5 \mathrm{~s}$ \\
\hline $\begin{array}{l}x^{10}-2 x^{9}+x^{8}-2 x^{7} \\
\quad+x^{6}-x^{5}-x^{2}-1\end{array}$ & 1.957362809 & $2.22214 \mathrm{e}-07$ & 22336604 & $29 \mathrm{~m} 7 \mathrm{~s}$ \\
\hline $\begin{array}{l}x^{10}-2 x^{9}+x^{7}-2 x^{6} \\
\quad+x^{4}-2 x^{3}+x-1\end{array}$ & 1.998277927 & $2.447 \mathrm{e}-08$ & 46943484 & $1 \mathrm{~h} 3 \mathrm{~m} 54 \mathrm{~s}$ \\
\hline
\end{tabular}

TABLE 4. Successful calculations with a spectrum over 20 million for $a(q)$.

\begin{tabular}{||l|c|c|c|c||}
\hline Pisot polynomial & $q$ & $a(q)$ & $\begin{array}{c}\text { Approximate } \\
\text { size of } \\
\text { spectrum in } \\
{\left[\alpha_{l}, \alpha_{u}\right]}\end{array}$ & $\begin{array}{c}\text { CPU } \\
\text { secs }\end{array}$ \\
\hline$x^{10}-x^{9}-x^{8}+x^{2}-1$ & 1.601755862 & $1.59445 \mathrm{e}-07$ & 33921896 & $30 \mathrm{~m} 38 \mathrm{~s}$ \\
$x^{10}-x^{9}-x^{8}-x^{2}+1$ & 1.632690733 & $1.03354 \mathrm{e}-07$ & 21835702 & $17 \mathrm{~m} 30 \mathrm{~s}$ \\
$x^{10}-2 x^{9}+x^{8}-x^{7}$ & 1.735143707 & $8.28149 \mathrm{e}-08$ & 32342934 & $29 \mathrm{~m} 18 \mathrm{~s}$ \\
$+x^{3}-x^{2}+x-1$ & & & & \\
\hline
\end{tabular}

Lastly given are two tables of the largest calculations done in the computation of $l(q)$ and $a(q)$. Any calculation which had an approximate spectrum size of over 20 million is listed. Due to the memory requirements of this program, any problem with a spectrum of approximate size over 48 million could not be computed. This accounts for 16 out of the 232 Pisot numbers of degree 10 or less. Table 3 is for $l(q)$, and Table 4 is for $a(q)$. 
The timings listed in these tables were done on a MIPS R10000 Processor Chip Revision: 3.4 (Main memory size: 4096 Mbytes). The code to perform these calculations was written in $\mathrm{C}++$, using the GNU compiler.

Precise values of $l(q)$ and $a(q)$ in terms of their polynomial evaluation at $q$ can be found on the web at 12 .

\section{Some Questions of $A(q)$ FOR NON-Pisot Numbers $q$}

Peres and Solomyak asked in [18] for which $q$ in $1<q<2$ is $A(q)$ dense. It was unknown to the authors then if there were any $q$ for which $q$ is not a Pisot number, yet $A(q)$ is not dense. Here, this is answered in the affirmative.

The examples of non-Pisot numbers $q$ where $A(q)$ is discrete required a search of 1868 possible candidates. To explain how these candidates were determined, consider the following theorem.

Theorem 5.1. If $q$ does not satisfy a polynomial of the form $\epsilon_{n} x^{n}+. .+\epsilon_{m} x^{m}+$ $\beta_{m-1} x^{m-1}+\cdots+\beta_{0}$ where $\epsilon_{i} \in\{ \pm 1\}$ and $\beta_{i} \in\{ \pm 2,0\}$, then $A(q)$ is not discrete.

Proof. Assume that $q$ does not satisfy such a polynomial. Take $P_{0}=1$. Then take $P_{n}=1-q \times P_{n-1}$ if $q \times P_{n-1}<1$ and $P_{n}=q \times P_{n-1}-1$ if $q \times P_{n-1}>1$. Clearly $P_{i} \in A(q)$ for all $i$ and $0 \leq P_{i} \leq 1$. If this sequence repeats, then $q$ satisfies the difference of the two \pm 1 polynomials in $q$, which is of the form described above, hence a contradiction. Hence, $\left\{P_{i}\right\}$ is an infinite nonrepeating sequence in $[0,1]$, and thus $A(q)$ is not discrete.

Corollary 1. If $q$ does not satisfy a height 2 polynomial, then $A(q)$ is not discrete.

Lemma 3. If $A(q)$ is discrete, then $A\left(q^{n}\right)$ is discrete for all $n$.

Proof. Let $\alpha=\sum_{i=1}^{m} \epsilon_{i} q^{n i}$ be a point in $A\left(q^{n}\right)$. Then $\left(q^{n-1}+q^{n-2}+\cdots+q+1\right) \alpha$ is in $A(q)$. Thus $\frac{q^{n}-1}{q-1} A\left(q^{n}\right) \subset A(q)$. Thus $A\left(q^{n}\right)$ is discrete.

Lemma 3, although of theoretical interest, is not of much practical use, as no $q<\sqrt{2}$, other than the two Pisot numbers, have been found where $A(q)$ is discrete.

With the limits imposed by Theorem 5.1 the search was restricted to the following:

1. All polynomials dividing a height 1 polynomial of degree $\leq 7$.

2. All polynomials dividing any \pm 1 polynomial up to degree 10 .

3. All polynomials dividing a polynomial of the form $\epsilon_{n} x^{n}+\epsilon_{n-1} x^{n-1}+\cdots \epsilon_{m} x^{m}$ $+\beta_{m-1} x^{m-1}+\cdots+\beta_{0}$ where $\epsilon_{i} \in\{ \pm 1\}$ and $\beta_{i} \in\{ \pm 2,0\}$ up to degree 7 .

Some observations that can be made on the basis of this search are as follows:

1. All examples of $q$ where $A(q)$ is discrete found are Perron numbers (all conjugates are of modulus less than $q$ ).

2. There were 120 examples found of non-Pisot numbers with discrete spectra $A(q)$.

3. There were 7 Salem numbers found (all but one conjugate is of modulus 1 ), with discrete spectra. They are given in Table 6

4. The only non-Pisot numbers $q$ whose minimal polynomial has Mahler measure less than 2 while $A(q)$ is discrete seems to be these Salem numbers.

5. The smallest (non-Pisot) number found with discrete spectrum is the Salem number 1.72208 of degree four (the root of $x^{4}-x^{3}-x^{2}-x+1$ ). 
6. The largest root of $x^{n}-x^{n-1}-\cdots-x+1$ is a Salem number with discrete spectrum (Theorem 5.3 and Theorem [5.4) and the only Salem numbers of degree 9 or less with discrete spectrum satisfy a polynomial of this type.

7. All $q$ found in the search of non-Pisot numbers satisfying a polynomial of the form $\epsilon_{n} x^{n}+\epsilon_{n-1} x^{n-1}+\cdots \epsilon_{m} x^{m}+\beta_{m-1} x^{m-1}+\cdots+\beta_{0}$ where $\epsilon_{i} \in\{ \pm 1\}$, $\beta_{i} \in\{ \pm 2,0\}$ and $m \geq 1$ do not have zero in the spectrum.

8. The smallest degree polynomial defining a $q$ such that $A(q)$ is discrete is $x^{3}-2 x-2$.

It is worth noting here the distinction between discrete spectra and uniformly discrete spectra. As will be shown, these examples found of non-Pisot numbers with discrete spectra $A(q)$ are "most probably" not uniformly discrete, and some provably not uniformly discrete.

Theorem 5.2. If $l(q)=0$, then $A(q)$ is not uniformly discrete, and if $A(q)$ is not uniformly discrete, then $l^{2}(q)=0$.

Proof. The first part follows by noticing that

$$
2 \Lambda(q)=\Lambda^{ \pm 2,0}(q) \subseteq A(q)-A(q)
$$

and the second part follows by noticing that

$$
A(q)-A(q) \subseteq \Lambda^{2}(q)
$$

So if the conjecture is true that $l(q)>0$ if and only if $q$ is Pisot, then we have that all of the non-Pisot numbers $q$ must have $A(q)$ nonuniformly discrete.

TABle 5. Polynomials with nonuniformly discrete spectrum, $A(q)$

\begin{tabular}{||l|l||}
\hline Non-Pisot polynomial & Root \\
\hline$x^{3}-2 x-2$ & 1.769292354 \\
$x^{4}-x^{3}-2 x-2$ & 1.873708564 \\
$x^{4}-2 x^{2}-2 x-2$ & 1.899321089 \\
$x^{5}-x^{4}-2 x^{2}-2$ & 1.803707279 \\
$x^{5}-x^{4}-x^{3}-2 x^{2}+2$ & 1.917514202 \\
$x^{5}-x^{4}-2 x^{2}-2 x-2$ & 1.942887561 \\
$x^{5}-2 x^{3}-2 x^{2}-2 x-2$ & 1.953501637 \\
$x^{6}-2 x^{4}-2 x^{3}-2$ & 1.813277575 \\
$x^{6}-x^{5}-x^{4}-2 x^{3}+2 x+2$ & 1.859080768 \\
$x^{6}-2 x^{4}-2 x^{3}-2 x^{2}+2$ & 1.865843123 \\
$x^{6}-x^{5}-x^{4}-2 x^{3}+2$ & 1.961038629 \\
$x^{6}-2 x^{4}-2 x^{3}-2 x^{2}-2 x-2$ & 1.977807115 \\
$x^{6}-x^{5}-x^{4}-x^{3}-2 x^{2}+2$ & 1.963984556 \\
$x^{7}-x^{6}-x^{5}-x^{4}+x^{3}-2 x^{2}+2$ & 1.815396315 \\
$x^{7}-x^{6}-x^{5}-2 x^{4}+2 x^{2}+2$ & 1.888840344 \\
$x^{7}-x^{6}-x^{5}-x^{4}-x^{3}+2$ & 1.903972308 \\
$x^{7}-x^{6}-x^{5}-2 x^{4}+2 x+2$ & 1.937730036 \\
$x^{7}-x^{6}-x^{5}-x^{4}-x^{3}-2 x^{2}+2 x+2$ & 1.945197233 \\
$x^{7}-x^{6}-x^{5}-2 x^{4}+2$ & 1.981204104 \\
$x^{7}-x^{6}-x^{5}-x^{4}-2 x^{3}+2$ & 1.982546502 \\
$x^{7}-x^{6}-x^{5}-x^{4}-x^{3}-2 x^{2}+2$ & 1.983151826 \\
\hline
\end{tabular}


The polynomials listed in Table 5 are known to have nonuniformly discrete spectra. These polynomials have discrete spectra, as a result of the search described earlier in this section. It is seen that $l(q)=0$ in all of the polynomials as they do not satisfy a height 1 polynomial [3, 10]. Thus by Theorem 5.2 these spectra are nonuniformly discrete spectra.

Next consider the Salem numbers in Table 6. By noticing that $1-2 x^{2}-3 x^{3}-$ $2 x^{4}+x^{6}$ divides $1-2 x+x^{2}-2 x^{3}+x^{4}-2 x^{5}+x^{6}-2 x^{7}+x^{8}$, and then $1-2 x+$ $x^{2}-\cdots-2 x^{2 n-1}+x^{2 n}$ divides $1-x-x^{2}-\cdots-x^{2 n}+x^{2 n+1}$, it can be noticed that all of the Salem numbers found with the property that $A(q)$ is discrete satisfy a polynomial of the form $1-x-x^{2}-\cdots-x^{n-1}+x^{n}$ for $n \geq 4$.

This led to the investigation of whether all of these numbers are Salem numbers, and if $A(q)$ is discrete in every case. (Both of these are answered in the affirmative below.) It is still unknown if this is all of the Salem numbers $q$ where $A(q)$ is discrete, and if these are the only such $q$ with Mahler measure less than 2 .

Theorem 5.3. The root of the polynomial $x^{n}-x^{n-1}-\cdots-x+1$ between 1 and 2 is a Salem number.

Proof. By [19] if $P(x)$ is a Pisot polynomial and $P^{*}(x)$ its reciprocal, then

$$
\left(x^{n} P(x)-P^{*}(x)\right) /(x-1)
$$

defines a Salem number provided it has a root greater than 1 . Here we take $P(x)=$ $x-2$. To see that there is a root between 0 and 1 , we use the intermediate value theorem.

Theorem 5.4. If $1<q$ satisfies the polynomial $1-x-x^{2}-\cdots-x^{n-1}+x^{n}$, then $A(q)$ is discrete.

TABLE 6. All Salem numbers of degree $\leq 10, q$ where the spectrum $A(q)$ is discrete.

\begin{tabular}{||l|l|l|l||}
\hline Salem polynomial & $q$ & $a(q)$ & $\begin{array}{l}\text { Approximate } \\
\text { size of } \\
\text { spectrum in } \\
{\left[\alpha_{l}, \alpha_{u}\right]}\end{array}$ \\
\hline \hline$x^{4}-x^{3}-x^{2}-x+1$ & 1.722083806 & 0.243489 & 11 \\
$x^{4}-2 x^{3}+x^{2}-2 x+1$ & 1.883203506 & 0.249038 & 13 \\
$x^{6}-x^{5}-x^{4}-x^{3}$ & 1.946856268 & 0.249814 & 15 \\
$-x^{2}-x+1$ & & & \\
$x^{6}-2 x^{5}+x^{4}-2 x^{3}$ & 1.974818708 & 0.249959 & 17 \\
$+x^{2}-2 x+1$ & & & \\
$x^{6}-2 x^{4}-3 x^{3}-2 x^{2}+1$ & 1.987793167 & 0.249991 & 19 \\
$x^{8}-2 x^{7}+x^{6}-2 x^{5}$ & 1.994004199 & 0.249998 & 21 \\
$+x^{4}-2 x^{3}+x^{2}$ & & & \\
$-2 x+1$ & & & \\
$x^{10}-x^{9}-x^{8}-x^{7}$ & 1.997032367 & 0.249999 & 23 \\
$-x^{6}-x^{5}-x^{4}$ & & & \\
$-x^{3}-x^{2}-x+1$ & & & \\
\hline
\end{tabular}


Proof. To see that $A(q)$ is discrete, simply consider the algorithm. The following observations are needed.

1. $q^{m}-q^{m-1}-\cdots-q+1>\frac{1}{q-1}$ for $m<n$.

2. $q^{n}-q^{n-1}-\cdots-q-1<\frac{-1}{q-1}$.

Thus at each step of the algorithm there is only one choice, and it must terminate after $n$ steps. (The case of polynomials with negative lead coefficients has been removed by symmetry.)

Thus it remains to prove these two observations.

1. First, notice that the roots $q_{n}$ of $q^{n}-q^{n-1}-\cdots-q+1$ form an increasing sequence bounded below by 1 and above by 2 . (This follows as $q_{n}^{n+1}-q_{n}^{n}-$ $\cdots-q_{n}+1=q_{n}^{n+1}-2 q_{n}^{n}=q_{n}^{n}\left(q_{n}-2\right)<0$ and $\left.2^{n+1}-2^{n}-\cdots-2+1=3>0.\right)$

Thus for $m \leq n-2$

$$
\begin{aligned}
q_{n}^{m+2}-q_{n}^{m+1}-\cdots-q_{n}+1 & \geq 0, \\
q_{n}^{m+2}-q_{n}^{m+1}-\cdots-q_{n}^{2} & \geq q_{n}-1, \\
q_{n}^{m}-q_{n}^{m-1}-\cdots-1 & \geq \frac{q_{n}-1}{q_{n}^{2}}, \\
q_{n}^{m}-q_{n}^{m-1}-\cdots-1 & >0, \\
q_{n}^{m}-q_{n}^{m-1}-\cdots+1 & >2, \\
q_{n}^{m}-q_{n}^{m-1}-\cdots+1 & >\frac{1}{q-1} .
\end{aligned}
$$

The last inequality follows as the smallest Salem number of this form is approximately 1.72 .

For $m=n-1$ and $q>\frac{3+\sqrt{17}}{4} \approx 1.780$ it follows that

$$
\begin{aligned}
0 & >-2 q^{2}+3 q+1, \\
0 & >\frac{q}{q-1}-2 q+1, \\
2 q-1 & >\frac{q}{q-1}, \\
q\left(q^{n-1}-\cdots-q+1\right) & >\frac{q}{q-1}, \\
q^{n-1}-\cdots-q+1 & >\frac{1}{q-1} .
\end{aligned}
$$

For the cases of $q<1.780$, we simply note that this is already covered as a special case in Table 6 .

2. Notice $q^{n}-q^{n-1}-\cdots-q-1=q^{n}-q^{n-1}-\cdots-q+1-2=-2<\frac{-1}{q-1}$.

From this, we deduce:

Corollary 2. For $q$ as above, $a(q)=\frac{q-1}{q^{2}} \approx \frac{1}{4}$.

\section{Finding \pm 1 POLYNomials With LLL}

If $q$ is not a Pisot number, yet is still an algebraic number, Algorithm 1 can be used to determine if $0 \in A(q)$. Unfortunately, for some $q$, this algorithm cannot return a negative answer and has the potential of returning no information at all. 
This is because it is not known if $A(q)$ is discrete; if $A(q)$ is not discrete, the algorithm is not guaranteed to terminate.

It is of interest to see which $q$ satisfy a \pm 1 polynomial, or equivalently when $0 \in A(q)$. For this, we introduce a few new algorithms, and a few observations.

Lemma 4. If the lead coefficient and tail coefficient of $p(x)$ are both odd, then $p(x) \mid 1+x+\cdots x^{n}(\bmod 2)$ for some $n$.

Proof. This follows from the observation that if $p(x)$ is irreducible modulo 2 , and of degree $n$, then $p(x) \mid x^{2^{n}-1}+1(\bmod 2)\left[13\right.$. Noticing that $\left(x^{n}+1\right)\left(x^{m}+1\right) /(x+1)=$ $x^{n+m-1}+1(\bmod 2)$ and $(x+1)\left(x^{n-1}+x^{n-2}+\cdots+x+1\right)=x^{n}+1(\bmod 2)$ completes the proof.

Algorithm 2 will for input $p(x)$ find a polynomial $q(x)$, such that $p(x) q(x)$ is of the form $1+x+\cdots+x^{n}(\bmod 2)$. Although it is possible to determine the value for $n$ in the lemma above by looking at the factorization of $p(x) \bmod 2$, it is easier to simply test each $n$ in order.

From here LLL can be often be used to find a polynomial $q^{\prime}(x)$ where $q^{\prime}(x) p(x)$ is a polynomial with \pm 1 coefficients. Recall if $L=\left\{\sum_{i=1}^{n} b_{i} v_{i}: b_{i} \in \mathbb{Z}\right\}$ is a lattice with a basis of vectors $\left\{v_{1}, \cdots, v_{n}\right\}$, then LLL will find a new basis for $L$ where each element of the basis will have a "small" norm. (We can choose any inner product for the lattice $L$.) For a good review of LLL see [4, 16, 17.

So, for a basis choose $p(x) q(x)$ as one basis element, and $2 p(x) x^{n}$ for $0 \leq n<$ $\operatorname{deg} q$ as the rest. It can be seen that if there is one basis element in the original basis with all odd coefficients, and all the rest have only even coefficients, then the resulting basis from LLL will have at least one basis element with only odd coefficients. Secondly, as LLL will try to minimize the sum of the squares of the coefficients (the norm we picked for this problem), this polynomial with odd coefficients will, with some luck, be a polynomial with \pm 1 coefficients.

This is written up into Algorithm 3.

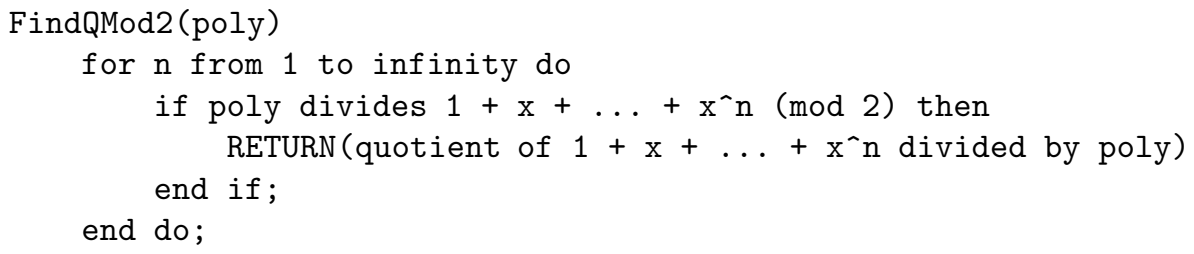

Algorithm 2: Finding a polynomial divisible by $p(x)(\bmod 2)$.

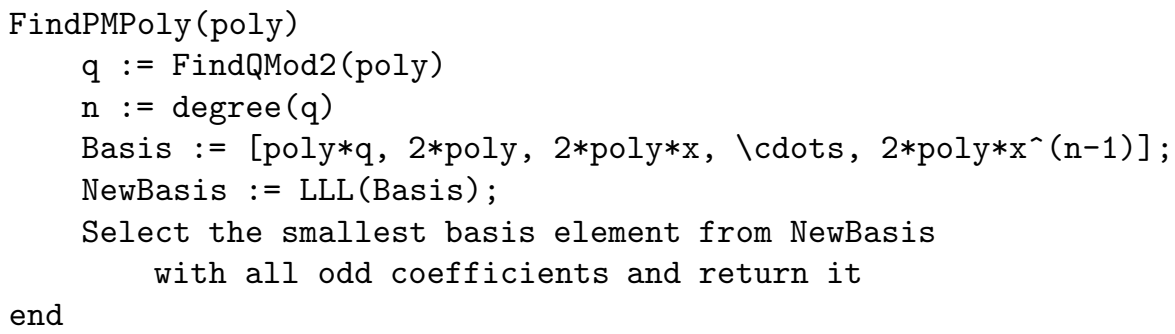

Algorithm 3: LLL to find a polynomial with small odd coefficients. 
TABle 7. Polynomials found by the LLL algorithm

\begin{tabular}{|c|c|}
\hline Pisot polynomial & \pm 1 polynomial it divides \\
\hline $\begin{array}{l}x^{2}-x-1 \\
x^{3}-x-1 \\
x^{3}-x^{2}-1 \\
x^{5}-x^{3}-x^{2}-x-1\end{array}$ & $\begin{array}{l}x^{2}-x-1 \\
x^{6}+x^{5}-x^{4}-x^{3}-x^{2}-x-1 \\
x^{6}-x^{5}+x^{4}-x^{3}-x^{2}-x-1 \\
-x^{30}+x^{29}+x^{28}-x^{27}+x^{26}+x^{25}-x^{24}-x^{23} \\
+x^{22}-x^{21}+x^{20}+x^{19}-x^{18}-x^{17}+x^{16}+x^{15} \\
+x^{14}-x^{13}-x^{12}+x^{11}-x^{10}+x^{9}+x^{8}-x^{7}-x^{6} \\
+x^{5}-x^{4}-x^{3}-x^{2}-x-1 \\
-x^{30}+x^{29}+x^{28}+x^{27}-x^{26}+x^{25}-x^{24}+x^{23} \\
+x^{22}-x^{21}-x^{20}+x^{19}-x^{18}-x^{17}-x^{16}+x^{15} \\
+x^{14}-x^{13}+x^{12}+x^{11}+x^{10}-x^{9}-x^{8}-x^{7}-x^{6} \\
-x^{5}-x^{4}-x^{3}-x^{2}+x+1\end{array}$ \\
\hline Salem polynomial & \pm 1 polynomial it divides \\
\hline $\begin{array}{l}x^{10}+x^{9}-x^{7}-x^{6}-x^{5} \\
-x^{4}-x^{3}+x+1 \\
x^{10}-x^{6}-x^{5}-x^{4}+1 \\
\\
x^{10}-x^{7}-x^{5}-x^{3}+1\end{array}$ & $\begin{array}{l}-x^{30}-x^{29}+x^{28}+x^{27}+x^{26}+x^{25}-x^{24}+x^{23} \\
-x^{22}-x^{21}-x^{20}+x^{19}+x^{18}+x^{17}-x^{16}-x^{15} \\
+x^{14}+x^{13}-x^{12}+x^{11}-x^{10}-x^{9}-x^{8}-x^{7}+x^{6} \\
+x^{5}+x^{4}+x^{3}+x^{2}-x-1 \\
-x^{30}-x^{29}+x^{28}+x^{27}+x^{26}+x^{25}+x^{24}-x^{23} \\
-x^{22}-x^{21}-x^{20}+x^{19}+x^{18}+x^{17}+x^{16}-x^{15} \\
-x^{14}-x^{13}-x^{12}+x^{11}-x^{10}+x^{9}+x^{8}-x^{7}+x^{6} \\
-x^{5}+x^{4}+x^{3}-x^{2}+x-1 \\
\text { Degree } 32 \text { example found. }\end{array}$ \\
\hline $\begin{array}{l}\text { Non-Pisot } \\
\text { Non-Salem polynomial }\end{array}$ & \pm 1 polynomial it divides \\
\hline $\begin{array}{l}x^{4}-x^{2}-1 \\
x^{6}-x^{4}-1 \\
x^{6}-x^{4}-x^{2}-1 \\
x^{4}+x^{3}-x^{2}-x-1 \\
x^{4}-x-1\end{array}$ & $\begin{array}{l}x^{5}+x^{4}-x^{3}-x^{2}-x-1 \\
x^{13}+x^{12}-x^{11}-x^{10}+x^{9}+x^{8} \\
-x^{7}-x^{6}-x^{5}-x^{4}-x^{3}-x^{2}-x-1 \\
x^{7}+x^{6}-x^{5}-x^{4}-x^{3}-x^{2}-x-1 \\
x^{4}+x^{3}-x^{2}-x-1 \\
x^{14}+x^{13}+x^{12}-x^{11}-x^{10} \\
-x^{9}-x^{8}-x^{7}-x^{6}-x^{5}+x^{4}-x^{3}-x^{2}-x-1\end{array}$ \\
\hline
\end{tabular}

Algorithm 3 experimentally works quite well for small problems. Unfortunately, for almost every polynomial of degree $n$, the resulting $q(x)$ is of degree $2^{n}-n$. This means that LLL must be performed on a basis of size $2^{n}-n$, which leads to an exponential time algorithm.

Some results are tabulated in Table 7 for Pisot numbers, Salem numbers, and non-Pisot non-Salem numbers.

\section{Conclusions}

There are still many questions which this paper leaves open:

1. Is the set of $q$ such that $a(q)=l(q)$ finite?

2. Does there exists and $\alpha \approx 1.95$ such that if $q<\alpha$, and $q$ Pisot, then $0 \in A(q)$ ?

3. Are all $q$ where $A(q)$ is discrete necessarily Perron?

4. Are the only $q$ where $A(q)$ is discrete and the Mahler measure of $q$ is less than 2 necessarily Salem numbers or Pisot numbers.

5. Do the only Salem numbers $q$ with $A(q)$ discrete satisfy $q^{n}-q^{n-1}-\cdots-q+1$ for some $n$ ? 
6. Is there a $\alpha \approx 1.72$ such that if $q<\alpha$ and $q$ is not Pisot, then $A(q)$ is not discrete?

7. Is it true that $l(q)>0$ if and only if $q$ is Pisot?

\section{REFERENCES}

1. David W. Boyd, Pisot and Salem numbers in intervals of the real line, Math. Comp. 32 (1978), 1244-1260. MR 58:10812

2. 96g:11090

3. Y. Bugeaud, On a property of Pisot numbers and related questions, Acta Math. Hungar. 73 (1996), 33-39. MR 98c:11113

4. Henri Cohen, A course in computational algebraic number theory, Graduate Texts in Mathematics, no. 138, Springer Verlag, New York, 1993. MR 94i:11105

5. Thomas H. Cormen, Charles E. Leiserson, and Ronald L. Rivest, Introduction to algorithms, MIT Press, Cambridge, MA, 1990. MR 91i:68001

6. P. Erdős, I. Joó, and F. J. Schnitzer, On Pisot numbers, Ann. Univ. Sci. Budapest. Eötvös Sect. Math. 39 (1996), 95-99 (1997). MR 98d:11127

7. P. Erdős, M. Joó, and I. Joó, On a problem of Tamás Varga, Bull. Soc. Math. France 120 (1992), 507-521. MR 93m:11076

8. P. Erdős and V. Komornik, Developments in non-integer bases, Acta Math. Hungar. 79 (1998), 57-83. MR 99e:11132

9. Pál Erdös, István Joó, and Vilmos Komornik, Characterization of the unique expansions $1=\sum_{i=1}^{\infty} q^{-n_{i}}$ and related problems, Bull. Soc. Math. France 118 (1990), 377-390. MR 91j:11006

10. Paul Erdős, István Joó, and Vilmos Komornik, On the sequence of numbers of the form $\epsilon_{0}+\epsilon_{1} q+\cdots+\epsilon_{n} q^{n}, \epsilon_{i} \in\{0,1\}$, Acta Arith. 83 (1998), 201-210. MR 99a:11022

11. Adriano M. Garsia, Arithmetic properties of Bernoulli convolutions, Trans. Amer. Math. Soc. 102 (1962), 409-432. MR 25:1409

12. Kevin Hare, Home page, http://www.cecm.sfu.ca/ ${ }^{\sim}$ kghare/PISOT/, 2000.

13. I.N. Herstein, Topics in algebra, second ed., John Wiley \& Sons, Toronto, 1975. MR 50:9456

14. Vilmos Komornik, Paola Loreti, and Marco Pedicini, An approximation property of Pisot numbers, J. Number Theory 80 (2000), 218-327. MR 2000k:11116

15. Ka-Sing Lau, Dimension of a family of singular Bernoulli convolutions, J. Funct. Anal. 116 (1993), 335-358. MR 95h:28013

16. A.K. Lenstra, H.W. Lenstra Jr, and L. Lovász, Factoring polynomials with rational coeffcients, Math. Ann. 261 (1982), 515-534. MR 84a:12002

17. Maurice Mignotte, Mathematics for computer algebra, Springer Verlag, New York, 1991. MR 92i:68071

18. Yuval Peres and Boris Solomyak, Approximation by polynomials with coefficients $\pm 1, \mathrm{~J}$. Number Theory 84 (2000), 185-198. CMP 2001:04

19. R. Salem, Power series with integral coefficients, Duke Math. J. 12 (1945), 153-172. MR 6:206b

Department of Mathematics and Statistics, Simon Fraser University, Burnaby, British Columbia, Canada V5A $1 \mathrm{~S} 6$

E-mail address: pborwein@math.sfu.ca

Department of Mathematics and Statistics, Simon Fraser University, Burnaby, British Columbia, Canada V5A $1 \mathrm{~S} 6$

E-mail address: kghare@cecm.math.sfu.ca 